\title{
Clinical/Scientific Notes
}

\section{Closure of a patent foramen ovale is associated with a decrease in prevalence of migraine}

Martijn C. Post, MD; Vincent Thijs, MD; Luc Herroelen, MD; and Werner I.H.L. Budts, MD, PhD

A patent foramen ovale ( $\mathrm{PFO}$ ) is one of the major causes of right-toleft shunt, and a causal relationship between migraine and a PFO has been suggested. ${ }^{1}$ We evaluated whether percutaneous closure of a PFO was associated with changes in the prevalence of migraine.

Methods. Patient selection. Patients with a PFO who had a paradoxical embolic event or systemic desaturation and who underwent a percutaneous closure in our center between February 1999 and September 2002 were included. The medical files were reviewed. The ethical committee approved the study.

Evaluation of migraine. A questionnaire was composed in such a way that a neurologist could diagnose migraine with or without aura (MA+ and MA-) according to the criteria of the International Headache Society. The questionnaire was sent to all patients and focused on three periods: 1 year before and 2 months and at least 6 months after percutaneous closure. Two neurologists blinded to the patients' files diagnosed MA+ and MA-.

Statistical analysis. Within-patient comparisons of the absence or presence of migraine were performed with the McNemar's paired $\chi^{2}$ test. Interobserver reliability was evaluated by measuring the kappa coefficient. $p$ Value $<0.05$ was considered significant. All statistical analyses were performed with GB Stat software (version 8.0; Dynamic Microsystems, Inc., Silver Spring, MD).

Results. Patient characteristics. Seventy-six patients (mean age, $50.7 \pm 12.9$ years) were selected, and 66 completed the questionnaire. In 57 patients, the period between PFO closure and completing the questionnaire was $>6$ months. The characteristics of patients who completed the questionnaire are summarized in the table.

Prevalence of migraine. The median time interval between the occurrence of a paradoxical embolic event and the closing procedure was 162 days (range, 0 to 3,613 days). The time between PFO closure and administration of the questionnaire was 579 days (range, 110 to 1,419 days).

Migraine was present in 26 of 66 patients (9 men and 17 women; 39.4\%). Twelve (18.2\%) had MA+, and $14(21.2 \%)$ had MA - . Two months after closure, the prevalence of MA+ and MAdecreased to $6.1 \%(4 / 66)$ and $6.1 \%(4 / 66 ; p<0.05$ vs before closure). At 6 months or more, the overall prevalence of migraine was $15.8 \%(9 / 57 ; p<0.05$ vs before closure). The prevalences of $\mathrm{MA}+$ and MA- were $5.3 \%(3 / 57 ; p<0.05$ vs before closure) and $10.5 \%(6 / 57 ; p=0.11$ vs before closure). The frequency of migraine attacks also decreased $(p<0.05)$. Seven patients were taking potential prophylactic migraine drugs 6 months after closure (six, $\beta$-blockers; one, calcium antagonists). The kappa coefficient for interobserver reliability for migraine was $0.8(p<0.05)$.

Discussion. Patients with migraine have a high prevalence of PFO. ${ }^{2}$ An increased rate of MA+ among stroke patients with PFO was found compared with patients with $\mathrm{PFO} .^{2} \mathrm{~A}$ causal relationship between PFO and migraine has been proposed. In individuals with a right-to-left shunt, a lower dose of venous trigger substances may be needed to induce migraine because the shunt permits the pulmonary filter to be bypassed. ${ }^{1}$ Moreover, the prevalence of migraine seems to decrease subsequent to PFO closure in patients with decompression illness. ${ }^{3}$

We evaluated whether PFO closure in patients who mainly had cryptogenic stroke would be associated with changes in the prevalence of migraine. We found a high rate of migraine in patients with PFO (39.4\%) and documented a significant and persistent decrease in prevalence of $\mathrm{MA}+\geq 6$ months after PFO closure. The frequency of migraine attacks also decreased significantly. Our data might fit with the recently reported experience that $\mathrm{MA}+$ decreased after PFO closure. ${ }^{4}$
Table Characteristics of patients who completed the questionnaire

\begin{tabular}{lccc}
\hline & Migraine & No migraine & $p$ \\
\hline No. of patients (n) & 26 & 40 & \\
Age (y, mean \pm SD) & $55 \pm 10$ & $53 \pm 12$ & 0.48 \\
Male/female & $9 / 17$ & $27 / 13$ & $<0.05$ \\
Indication PFO closure & & & \\
$\quad$ Stroke (n) & 24 & 37 & 0.94 \\
Peripheral embolism (n) & 2 & 1 & 0.33 \\
Brain abscesses (n) & 0 & 1 & \\
Desaturation (n) & 0 & 1 &
\end{tabular}

Frequency of migraine attacks prior to closure

Almost monthly (n)

Almost weekly (n)

Several times a week (n) 8

$\mathrm{PFO}=$ patent foramen ovale

The prevalence of migraine also decreases with age; however, we believe that the changes in our study are too pronounced to be explained by the natural history of migraine. ${ }^{5}$ Most of our patients were treated with low-dose aspirin, which could also influence migraine prophylaxis. The effect of lowdose aspirin, if any, seems to be modest. ${ }^{6}$ The placebo effect in migraine therapy is potent, but the decrease in prevalence of migraine in our study seems to be larger than reported placebo effect rates of 20 to $40 \%$. $^{7}$

Nevertheless, this study has important limitations. It is a retrospective, nonrandomized trial of patients selected from a hospital-based database. The questionnaire may be influenced by recall bias.

The prevalence of migraine in patients with a $\mathrm{PFO}$ is high. After $\geq 6$ months, percutaneous PFO closure is associated with a decrease in the prevalence of MA+. Whether percutaneous PFO closure has the potential to manage migraine needs to be determined in a prospective randomized trial.

From the Department of Internal Medicine, Division of Cardiology (Drs. Post and Budts), and Department of Neurology (Drs. Thijs and Herroelen), University Hospital Gasthuisberg, Leuven, Belgium.

Received September 15, 2003. Accepted in final form November 13, 2003.

Address correspondence and reprint requests to Dr. Werner Budts, Division of Cardiology, University Hospital Gasthuisberg, Herestraat 49, 3000 Leuven, Belgium; e-mail: Werner.Budts@uz.kuleuven.ac.be

Copyright @ 2004 by AAN Enterprises, Inc.

\section{References}

1. Wilmshurst P, Nightingale S. Relationship between migraine and cardiac and pulmonary right-to-left shunts. Clin Sci (Lond) 2001;100:215-220.

2. Sztajzel R, Genoud D, Roth S, Mermillod B, Le Floch-Rohr J. Patent foramen ovale, a possible cause of symptomatic migraine: a study of 74 patients with acute ischemic stroke. Cerebrovasc Dis 2002;13:102-106.

3. Wilmshurst PT, Nightingale S, Walsh KP, Morrison WL. Effect on migraine of closure of cardiac right-to-left shunts to prevent recurrence of decompression illness or stroke or for haemodynamic reasons. Lancet 2000;356:1648-1651. 
4. Morandi E, Anzola GP, Angeli S, Melzi G, Onorato E. Transcatheter closure of patent foramen ovale: a new migraine treatment? J Interv Cardiol 2003;16:39-42.

5. Henry P, Auray JP, Gaudin AF, et al. Prevalence and clinical characteristics of migraine in France. Neurology 2002;59:232-237.
6. Bensenor IM, Cook NR, Lee IM, Chown MJ, Hennekens CH, Buring JE. Low-dose aspirin for migraine prophylaxis in women. Cephalalgia 2001; 21:175-183.

7. van der Kuy PH, Lohman JJ. A quantification of the placebo response in migraine prophylaxis. Cephalalgia 2002;22:265-270.

\section{Prolactinoma presenting as painful postganglionic Horner syndrome}

\section{A.V. Talkad, MD; J.C. Kattah, MD; M.Y. Xu, MD, PhD;}

E.H. Orth, DO; and J.Y. Chang, $M D$

Painful postganglionic Horner syndrome may be the initial presentation of an internal carotid artery (ICA) dissection and less frequently is a complication of cluster headache. ${ }^{1}$ However, it is not a frequent presentation of pituitary adenoma. We describe a patient with prolactinoma that initially manifested with painful Horner syndrome and improved on dopaminergic therapy. Eighteen months after treatment was discontinued, the patient sought treatment for a cavernous sinus syndrome that responded well to high-dose dopaminergic therapy.

Case report. A 52-year-old man was examined by one of our staff neurologists for an intermittent right hemicrania, retro-orbital pain and a narrowed palpebral fissure in March 1999. He had no history of alcoholism, head trauma, hypertension, diabetes mellitus, chronic headaches, impotence, or decreased libido. He smokes one pack of cigarettes daily. Examination revealed a 2-mm ptosis of the right upper eyelid and a $2-\mathrm{mm}$ pupil that failed to dilate in the dark and after hydroxyamphetamine. The left pupil, in contrast, measured $3 \mathrm{~mm}$ and dilated to $5 \mathrm{~mm}$ in the dark and to $8 \mathrm{~mm}$ after hydroxyamphetamine. Both pupils reacted to light and accommodation. Visual acuity $\left(\mathrm{V}_{\mathrm{ac}}\right)$, color vision, visual fields, funduscopy, and remaining physical examination were normal. Brain MRI showed a discrete mass surrounding the carotid artery (figure, left). Serum prolactin level was elevated at $566.4 \mathrm{ng} / \mathrm{mL}$ (normal range 0.5 to $18.1 \mathrm{ng} / \mathrm{mL}$ ). Bromocriptine $5 \mathrm{mg} / \mathrm{d}$ was initiated, and the patient experienced symptomatic improvement during the ensuing 6 months.

Thirty-two months later, he again sought treatment at our institution for worsening right-sided headache and diplopia. Eighteen months earlier, the patient decided to discontinue his bromocriptine. During the interval, he had no change in his history. Examination revealed a 4-prism diopter right exophoria and a partial right oculomotor nerve paresis with partial ptosis of the upper eyelid; symmetric limitation of infraduction, supraduction, and adduction; and a 4-mm pupil with preservation of pupillary responses. Additionally, there was complete right sixth nerve palsy with hypesthesia in the right $\mathrm{V}_{1}$ and $\mathrm{V}_{2}$ dermatomes. Color vision, funduscopy, visual fields, and $\mathrm{V}_{\mathrm{ac}}$ were normal bilaterally. During the next 2 days, he developed a complete ophthalmoplegia of the right eye.

Brain MRI showed a $2.2 \times 1.8 \times 3$-cm lesion filling the right cavernous sinus adjacent to, but not compressing, the optic chiasm (figure, middle). The tumor now encircled the cavernous portion of the right ICA. Serum prolactin at that time was $281.3 \mathrm{ng} / \mathrm{mL}$. The patient was started on bromocriptine $15 \mathrm{mg}$ daily and cabergoline $1 \mathrm{mg}$ twice weekly. Ten days after cabergoline was added, serum prolactin level decreased to $22.1 \mathrm{ng} / \mathrm{mL}$.

Four months later, follow-up MRI showed a 50\% reduction in tumor bulk with the tumor still encircling the cavernous right ICA (figure, right). Clinically, he had resolution of the Horner syndrome and third and sixth nerve palsies, and return of facial sensation. In addition, there were no signs of aberrant regeneration. The patient was able to remain compliant with dual dopamine agonist therapy, except for nausea managed with ondansetron.

Discussion. A pituitary adenoma, presenting as a focal mass in the cavernous sinus, without diffuse sellar involvement, is distinctly uncommon. We are unaware of previous reports of pituitary tumors manifesting initially as postganglionic Horner syndrome. Although our patient did not have symptoms of hyperprolactinemia, a markedly increased prolactin level directed us to this diagnostic possibility. The successful effect of dopaminergic therapy, with resolution of the presenting symptoms and signs and virtual normalization of the serum prolactin level, left no doubt as to the diagnosis.

Prolactinoma is not initially thought of as a cause of an isolated involvement of postganglionic sympathetic fibers. Raeder ${ }^{2}$ first reported simultaneous impairment of sympathetic and trigeminal nerve fibers in a patient with an extrinsic mass lateral to the cavernous sinus. Our patient mimicked this presentation with a mass intrinsic to the cavernous sinus. Unlike his case, there was no involvement of motor trigeminal fibers.

Our patient had already been treated successfully with a single dopamine agonist but now presented with cavernous sinus syndrome after discontinuing therapy for 18 months. ${ }^{3}$ The main impetus to try simultaneous, high-dose dopamine agonists was to elicit a rapid response given the severity of the pain and ophthalmoplegia and to rule out the possibility of a prolactinsecreting carcinoma. The remarkable clinical and imaging response to dual dopamine agonists essentially served to elucidate the benign neuroendocrine character of the tumor, allowing a diagnosis without need for tissue biopsy or malignant tissue markers. ${ }^{4}$

From the Departments of Neurology (Drs. Talkad, Kattah, and Xu) and Internal Medicine (Drs. Orth and Chang), University of Illinois, Peoria, IL.

Received July 24, 2003. Accepted in final form September 8, 2003.

Address correspondence and reprint requests Dr. Arun Talkad, University of Illinois at Peoria, OSF St. Francis Medical Center, Department of Neurology, 530 NE Glen Oak Avenue, Peoria, IL 61637; e-mail: talkadmd@uic.edu

Copyright $\odot 2004$ by AAN Enterprises, Inc.
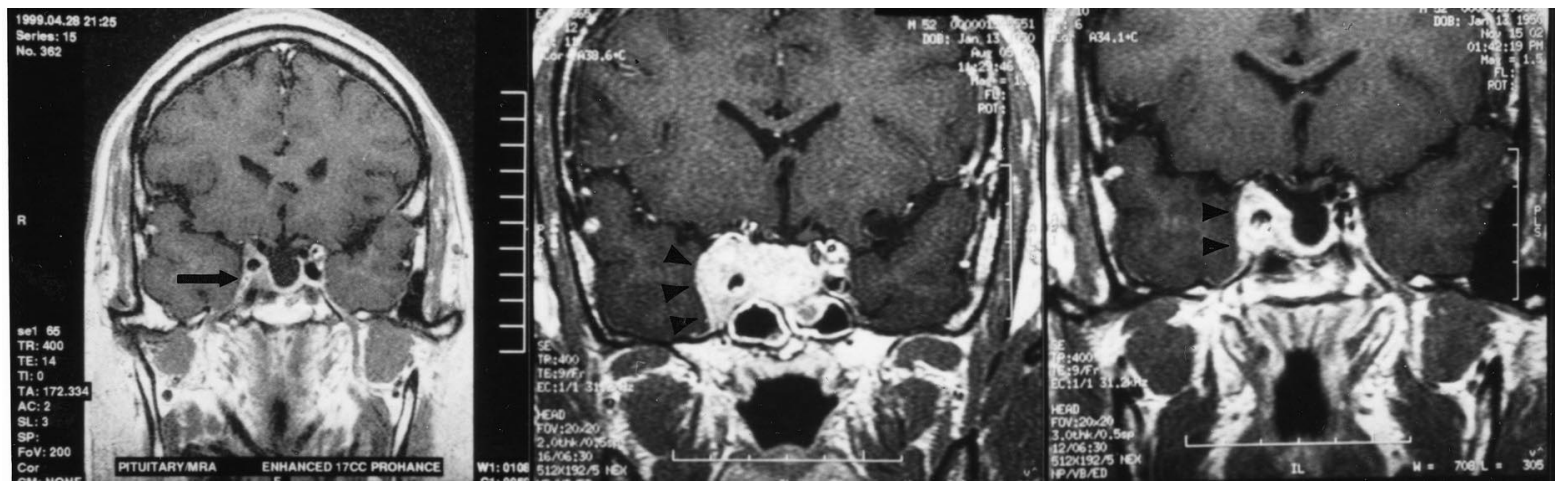

Figure. T1-weighted, contrast-enhanced MRI of brain. (Left) April 1999, performed after initial visit. (Middle) August 2002, 1 month after starting dual agonist therapy. (Right) November 2002, only minimal symptoms present with reported $50 \%$ reduction radiographically in tumor bulk. 


\section{References}

1. Cintron R, Kattah JC. Oculosympathetic paresis and hemicrania in spontaneous dissection of the internal carotid artery. Neuroophthalmology 1995;15:241-248.

2. Raeder JG. Paratrigeminal paralysis of oculopupillary sympathetics. Brain 1924;47:149-158.
3. Liuzzi A, Dallabonzana D, Oppizzi G, et al. Low doses of dopamine agonists in the long-term treatment of macroprolactinomas. N Engl J Med 1985;313:656.

4. Atienza DM, Vigersky RJ, Lack EE, et al. Prolactin-producing pituitary carcinoma with pulmonary metastasis. Cancer 1991;68: $1605-1610$

\section{IV insulin during acute cerebral infarction in diabetic patients}

Askiel Bruno, MD; Chandan Saha, PhD; Linda S. Williams, MD; and Ravi Shankar, $M D$

Although hyperglycemia can be a nonspecific response to stress, tight glycemic control during acute myocardial infarction ${ }^{1}$ and in postoperative ventilated patients ${ }^{2}$ has been shown to significantly reduce mortality. Animal ${ }^{3}$ and human ${ }^{4-6}$ studies suggest that hyperglycemia also augments acute cerebral ischemia, and clinical efficacy of tight glycemic control during acute cerebral infarction is being investigated. ${ }^{7}$ Good glycemic control during acute cerebral infarction in nondiabetic patients appears feasible and safe, but this has not been reported in patients with diabetes mellitus. ${ }^{7}$

Methods. Our Institutional Review Board approved this study, and all subjects signed a valid informed consent. We prospectively administered an IV insulin protocol initiated within 12 hours after onset of cerebral infarction to 24 consecutive patients with admission hyperglycemia ( 9.4 to $22.2 \mathrm{mmol} / \mathrm{L}$ or 170 to 400 $\mathrm{mg} / \mathrm{dL}$ ). Nurses supervised closely by the principal investigator administered the insulin protocol in an open-label fashion.

We modified our insulin protocol after each three to seven treated subjects to improve glycemic control and minimize hypoglycemia while keeping the protocol practical. We adjusted the insulin infusion rate every 1 to 2 hours if needed based on capillary glucose measurements to maintain the glucose between 3.9 and $7.2 \mathrm{mmol} / \mathrm{L}$ (or 70 to $130 \mathrm{mg} / \mathrm{dL}$ ). Table E-1 shows our initial and latest insulin protocols (see table E-1 on the Neurology Web site). Subjects able to eat received diabetic diets, along with subcutaneous insulin $0.12 \mathrm{U} / \mathrm{kg}$ before each meal. We continued the insulin protocol for up to 72 hours (range, 17 to 72 hours; mean, 54 hours). Oral antidiabetic medications were discontinued during insulin infusion.

We defined hypoglycemia as $<3.0 \mathrm{mmol} / \mathrm{L}$ ( or $<54 \mathrm{mg} / \mathrm{dL}$ ) and recurrent hyperglycemia as $>9.4 \mathrm{mmol} / \mathrm{L}$ (or $>170 \mathrm{mg} / \mathrm{dL}$ ) after the glucose had been lowered into the target range, and determined which episodes of glucose excursion were associated with potentially contributory protocol deviations.

Results. Fourteen women and 10 men were enrolled with a mean age of $67 \pm 11$ years. History of diabetes mellitus type 2 was present in $21(88 \%)$ subjects, and the remaining 3 were newly

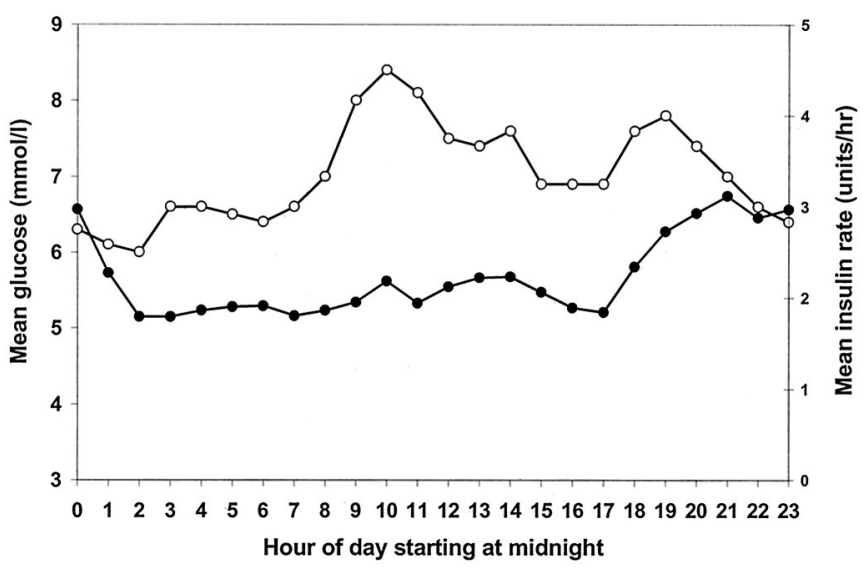

Figure. Mean glucose levels and insulin infusion rates in all 24 subjects during the entire treatment period synchronized to one 24-hour period. $\mathrm{O}=$ mean glucose; $\mathbf{0}=$ insulin rate. diagnosed with it during hospitalization. The median admission NIH Stroke Scale (NIHSS) score was 6 (range, 1 to 25).

In the 24 subjects the mean admission glucose was $14.7 \pm 4.9$ $\mathrm{mmol} / \mathrm{L}$ (or $264 \pm 88 \mathrm{mg} / \mathrm{dL}$ ), and during insulin infusion it was $7.3 \pm 1.1 \mathrm{mmol} / \mathrm{L}$ (or $131 \pm 20 \mathrm{mg} / \mathrm{dL}$ ). The mean glucose level reached the target range 5 hours after starting insulin infusion. Three peaks can be seen (figure) in the daily mean glucose level during the 10 th (10 to $11 \mathrm{AM}), 14$ th (2 to $3 \mathrm{PM})$, and 19 th ( 7 to $8 \mathrm{PM}$ ) hours corresponding to three daily meals normally given to patients. Many subjects in this study resumed eating on the second hospital day.

Table E-2 shows all the protocol deviations that contributed to hypoglycemia and recurrent hyperglycemia (see table E-2 on the Neurology Web site). At least one episode of hypoglycemia occurred in $11(46 \%)$ subjects, and protocol deviations accounted for 3 of 19 (16\%) episodes. Symptomatic hypoglycemia occurred in five (21\%) subjects and included adrenergic manifestations in all five and lethargy in one. All episodes of hypoglycemia were rapidly detected, and all symptoms resolved completely within 30 minutes after stopping insulin and giving concentrated glucose. The hypoglycemia episodes were not temporally related to neurologic deteriorations. Recurrent hyperglycemia occurred in $21(88 \%)$ subjects, and protocol deviations accounted for 9 of 48 (19\%) episodes.

Seventeen $(70.8 \%)$ subjects remained stable or improved continuously; four (16.7\%) had neurologic deterioration (increase on the NIHSS by $\geq 2$ points) soon after admission and then improved; and three $(12.5 \%)$ with severe strokes (admission NIHSS, 18 to 25) died from stroke complications.

Discussion. Our insulin protocol, although still suboptimal, represents a reasonable combination of simplicity, safety, and glycemic control. Given the dynamic nature of cell death during acute cerebral infarction, it is important to note that the glucose level was lowered into the target range within 5 hours after starting insulin.

Postprandial hyperglycemia appears to be a substantial contributor to recurrent hyperglycemia. Individualized doses of rapidly acting subcutaneous insulin, given right after meals, should reduce postprandial hyperglycemia within our protocol. Protocol deviations can result in recurrent hyperglycemia and hypoglycemia. The deviations will likely be minimized if the protocol is kept simple and used frequently. Three revisions may further limit hypoglycemia: 1) higher frequency of glucose monitoring or continuous monitoring; ${ }^{6} 2$ ) including glucose in the insulin solution, as was done in other studies: ${ }^{1,7}$ and 3) using a higher target range.

Good glycemic control during acute cerebral infarction in patients with diabetes mellitus type 2 is feasible and appears relatively safe. We plan to make additional modifications to our insulin protocol during a pilot clinical trial that is in progress. In this pilot trial, we will evaluate the feasibility of randomized, blinded treatment with IV (aggressive) vs subcutaneous (usual care) insulin in patients with acute cerebral infarction and admission hyperglycemia.

Additional material related to this article can be found on the Neurology Web site. Go to www.neurology.org and scroll down the Table of Contents for the April 27 issue to find the title link for this article.

From the Departments of Neurology (Drs. Bruno and Williams), Medicine (Dr. Saha), and Pediatrics (Dr. Shankar), Indiana University School of Medicine, Indianapolis, IN.

Received October 8, 2003. Accepted in final form November 26, 2003.

Address correspondence and reprint requests to Dr. Askiel Bruno, Indiana University School of Medicine, Department of Neurology, 541 Clinical Drive, RM 290C, Indianapolis, IN 46202-5111; e-mail: abruno@iupui.edu

Copyright (C) 2004 by AAN Enterprises, Inc. 


\section{References}

1. Malmberg K, for the DIGAMI (Diabetes Mellitus Insulin Glucose Infusion in Acute Myocardial Infarction) Study Group. Prospective randomised study of intensive insulin treatment on long term survival after acute myocardial infarction in patients with diabetes mellitus. BMJ 1997;314:1512-1515.

2. van den Berghe $\mathrm{G}$, Wouters $\mathrm{P}$, Weekers $\mathrm{F}$, et al. Intensive insulin therapy in critically ill patients. N Engl J Med 2001;345:1359-1367.

3. Kent TA, Soukup VM, Fabian RH. Heterogeneity affecting outcome from acute stroke therapy: making reperfusion worse. Stroke 2001;32:2318-2327.

4. Williams LS, Rotich J, Qi R, et al. Effects of admission hyperglycemia on mortality and costs in acute ischemic stroke. Neurology 2002;59:67-71.

5. Bruno A, Levine SR, Frankel MR, et al. Admission glucose level and clinical outcomes in the NINDS rt-PA Stroke Trial. Neurology 2002;59: 669-674.

6. Baird TA, Parsons MW, Phanh T, et al. Persistent poststroke hyperglycemia is independently associated with infarct expansion and worse clinical outcome. Stroke 2003;34:2208-2214.

7. Scott JF, Robinson GM, French JM, et al. Glucose potassium insulin infusions in the treatment of acute stroke patients with mild to moderate hyperglycemia: the Glucose Insulin in Stroke Trial (GIST). Stroke 1999;30:793-799.

\section{Sudden unilateral deafness due to a right vertebral artery dissection}

S.F.A. Raupp, MD; K. Jellema, MD; M. Sluzewski, MD, PhD; P.L.M. de Kort, $M D, P h D$; and L.H. Visser, $M D, P h D$

Case report. We present a 42-year-old woman without a known connective tissue disorder, who sought treatment at the emergency department because of sudden right-sided deafness, vertigo, and tendency to fall to the right. She also reported right-sided neck pain for 3 days.

Neurologic examination, performed 1 day after the onset of symptoms, revealed total deafness on the right side. There were no other abnormalities, including no nystagmus (also not present after use of Frenzel glasses) and no ataxia.

An MRI scan showed a small cerebellar infarction in the territory of the right posterior inferior cerebellar artery (PICA) on the T2-weighted image (figure, A). On the sagittal T1-weighted images, the distal part of the right vertebral artery was hyperintense, and no flow void was seen (not shown). A contrast-enhanced MR angiography showed a normal proximal right vertebral artery that showed irregularities and tapering in the high cervical portion. The distal portion of the right vertebral artery was occluded (figure, B and C).

The combination of image findings suggest vertebral dissection.

In this patient, sudden deafness, vertigo, and neck pain were caused by a spontaneous right vertebral artery dissection. The patient was treated with low molecular weight heparin, especially to prevent new neurologic signs and symptoms. During follow-up evaluation, the unilateral deafness persisted without any other sequelae.

The differential diagnosis of sudden deafness includes trauma, viral disease, syphilis, Lyme disease, vascular disease, HIV, ototoxic drugs, autoimmune inner ear disease, perilymph fistulae, Meniere disease, and acoustic neurinoma. ${ }^{1-3}$

The symptoms of sudden deafness combined with vertigo and neck pain suggested the diagnosis of a vertebral artery dissection.

Discussion. Sudden deafness has been described in anterior inferior cerebellar artery (AICA) infarction. Lee et al. described the signs and symptoms of 12 patients with unilateral AICA in- farction. ${ }^{1}$ Vertigo was the initial symptom in all patients, accompanied by a horizontal-rotatory nystagmus beating toward the healthy side. Sensorineural hearing loss was present in 11 patients. Unlike our patient, all 12 patients had gait and limb ataxia caused by involvement of the middle cerebellar peduncle or anterior inferior cerebellum. ${ }^{1}$

Another recent article described bilateral deafness in vertebral insufficiency caused by stenosis of the basilar artery. Vertigo was present, but no nystagmus was observed in this patient as well. ${ }^{4}$

Our patient had unilateral sudden deafness caused by a right vertebral dissection. In most patients, the internal auditory artery originates from the AICA, but in a few, it branches off the PICA. In our patient, most likely, small emboli originating from the dissection caused a small selective cerebellar and cochlear infarction, sparing the vestibular apparatus. This led to unilateral sensorineural hearing loss without nystagmus. In conclusion, for patients presenting with neck pain and sudden unilateral sensorineural deafness, with or without vertigo, a vertebral artery dissection should be considered and MR angiography performed.

From the Department of Neurology and Radiology, St. Elisabeth Hospital, Tilburg, The Netherlands.

Received September 15, 2003. Accepted in final form December 1, 2003.

Address correspondence and reprint requests to Dr. Sigrid Raupp, Department of Neurology and Radiology, St. Elisabeth Hospital, Hilvarenbeekseweg 60, Tilburg, The Netherlands; e-mail: sigridraupp@hotmail.com

Copyright @ 2004 by AAN Enterprises, Inc.

\section{References}

1. Lee H, Sohn SI, Jung DK, et al. Sudden deafness and anterior inferior cerebellar artery infarction. Stroke 2002;33:2807-2812.

2. Cadoni G, Fetoni AR, Agostino S, et al. Autoimmunity in sudden sensorineural hearing loss: possible role of anti-endothelial cell autoantibodies. Acta Otolaryngol Suppl 2002;548:30-33.

3. Fitzgerald DC, Mark AS. Sudden hearing loss: frequency of abnormal findings on contrast-enhanced MR studies. AJNR Am J Neuroradiol 1998;19:1433-1436.

4. Lee H, Yi HA, Baloh HW. Sudden bilateral simultaneous deafness with vertigo as a sole manifestation of vertebrobasilar insufficiency. J Neurol Neurosurg Psychiatry 2003;74:539-541.
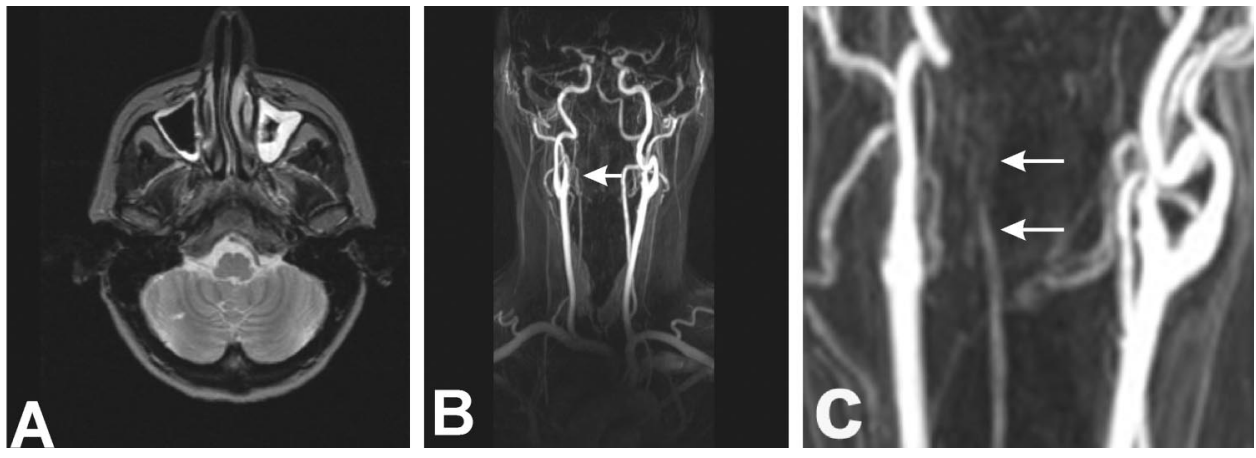

Figure. (A) MRI (T2-weighted) showing a small right-sided posterior inferior cerebellar artery (PICA) infarction. ( $B$ and $C$ ) Contrast-enhanced $M R$ angiography showing a normal proximal right vertebral artery with irregularities and tapering in the high cervical portion. 


\section{Plasmapheresis improves outcome in postinfectious cerebellitis induced by Epstein-Barr virus}

Jeremy D. Schmahmann, MD

Postinfectious cerebellitis (PIC) is a rare complication of EpsteinBarr virus (EBV) infection. Whereas its course is usually regarded as self-limited, it can be prolonged and produce considerable residual disability. We report two adults with persistent PIC after EBV infection who demonstrated complete and rapid recovery with a course of plasmapheresis (total plasma exchange [TPE]).

Case reports. Case 1. A 20-year-old woman (Case 15 in reference 1) developed fever and myalgia, followed 2 weeks later by onset over 3 days of slurred speech, gait instability, and difficulty judging distances. Examination revealed hypermetric saccadic eye movements, cerebellar dysarthria, dysmetria, dysdiadochokinesis, and ataxic gait. She had flattened affect, decreased verbal output and phonemic fluency, perseveration, and poor visual-spatial planning. Results of strength, reflex, and sensory testing were normal. Gadolinium-enhanced brain MRI and EEG were normal. CSF revealed three lymphocytes, normal protein, and glucose. Heterophile antibody was positive; serum EBV titer was 1:896; EBV viral capsid was 1:1280; restricted immunoglobulin (Ig) G was 1:320; and EBV nuclear antigen (EBNA) was 1:20, diagnostic of chronic or recurrent EBV infection. CSF viral capsid, diffuse and restricted IgG, and EBNA were normal. The persistence of motor and cognitive symptoms at 1 month prompted treatment with plasmapheresis. There was marked improvement in a stepwise fashion the day after each of six sessions of TPE. One week after treatment, the examination was only minimally abnormal, and 1 month after treatment, all cerebellar motor tests and neuropsychological tests had returned to normal, except for tests of complex and unstructured list learning.

Case 2. A 28-year-old man developed fever, myalgia, and pharyngitis. Two to 3 weeks later he had vertigo, ataxia, dysarthria, untidy handwriting, and incoordination of his limbs. He had difficulty concentrating and making quick decisions, and his family noted a flattened affect. Brain MRI and EEG were normal. EBV IgG titer was 1:640; IgM was elevated (1/10); and EBNA was negative, consistent with primary infection $<6$ weeks previously. Symptoms persisted, and examination 3 months after the onset of the illness revealed hypermetric saccades, cerebellar dysarthria, upper extremity rebound, dysmetria and dysdiadochokinesis, widened stance, and ataxic gait. Strength, reflexes, and sensation were normal. Attention, declarative learning, and phonemic fluency were reduced; thinking was concrete; and he perseverated on the Luria diagram. This lack of recovery prompted treatment with plasmapheresis. During the course of five TPE sessions, the cerebellar motor syndrome abruptly improved, and thinking and personality reverted to the premorbid state. Three months after TPE, the examination was normal.

Discussion. Acute cerebellar meningoencephalitis from EBV infection is rare, occurring more commonly in children than in adults. It is characterized by an acute pancerebellar syndrome sometimes with brainstem signs or diffuse encephalitis, meningis- mus, CSF pleocytosis, positive serologic tests for EBV in the CSF, and MRI abnormalities.

In contrast, PIC is thought to be an immune-mediated response to viral infection occurring 1 to 3 weeks later (range, 1 to 43 days $^{2}$ ), characterized by ataxia and dysmetria ${ }^{2}$ with minimal CSF leukocytosis, no systemic features, and negative CSF EBV titers despite positive serum titers. Neuropsychiatric features are also observed. ${ }^{1,2}$ MRI is usually normal, but SPECT scans may show decreased ${ }^{3}$ or increased perfusion. ${ }^{4}$

Reports generally stress the benign nature of postinfectious cerebellar ataxia, ${ }^{5}$ but Connolly et al. ${ }^{2}$ found that residual motor and cognitive deficits persisted for months or years, and in some cases deficits were permanent. Persistent cerebellar ataxia has also been noted in adults. ${ }^{6}$

Both our patients remained prominently compromised 1 month and 3 months after the onset of symptoms, and this time course, together with serologic tests in both patients and CSF analysis in Case 1, supported the diagnosis of EBV PIC. Continued clinical disability prompted the decision to treat. Recovery was rapid, complete, and occurred within days of the full course of conventional TPE (five to six exchanges), indicating a cause-and-effect relationship.

Successful improvement of PIC with IV Ig has been noted, but to our knowledge there are no published accounts of the use of TPE in this disorder., ${ }^{47}$ The success of TPE in our patients suggests that immune-modulating therapy may limit the duration of illness and potential long-term motor and cognitive deficits in patients with PIC from EBV. Prospective clinical trials could address this possibility more rigorously. The rapid recovery of PIC with TPE 3 months into the illness also implies that there is a protracted stage of antibody-mediated, reversible neuronal toxicity before Purkinje cell death.

From the Department of Neurology, Massachusetts General Hospital and Harvard Medical School, Boston, MA

Received September 6, 2003. Accepted in final form December 10, 2003.

Address correspondence and reprint requests to Dr. Jeremy D. Schmahmann, Department of Neurology, VBK 915, Massachusetts General Hospital Fruit Street, Boston, MA 02114; e-mail: jschmahmann@partners.org

Copyright (C) 2004 by AAN Enterprises, Inc.

\section{References}

1. Schmahmann JD, Sherman J. The cerebellar cognitive affective syndrome. Brain 1998;121:561-579.

2. Connolly AM, Dodson WE, Prensky AL, Rust RS. Course and outcome of acute cerebellar ataxia. Ann Neurol 1994;35:673-679.

3. San Pedro EC, Mountz JM, Liu HG, Deutsch G. Postinfectious cerebellitis: clinical significance of Tc-99m HMPAO brain SPECT compared with MRI. Clin Nucl Med 1998;23:212-216.

4. Daaboul Y, Vern BA, Blend MJ. Brain SPECT imaging and treatment with IVIg in acute post-infectious cerebellar ataxia: case report. Neurol Res 1998;20:85-88.

5. Nussinovitch M, Prais D, Volovitz B, Shapiro R, Amir J. Post-infectious acute cerebellar ataxia in children. Clin Pediatr (Phila) 2003;42:581-584.

6. Klockgether T, Doller G, Wullner U, Petersen D, Dichgans J. Cerebellar encephalitis in adults. J Neurol 1993;240:17-20.

7. Go T. Intravenous immunoglobulin therapy for acute cerebellar ataxia. Acta Paediatr 2003;92:504-506.

\section{Insulinoma misdiagnosed as intractable epilepsy}

M.-H. Dion, MD; P. Cossette, MD, MSc; J.-M. St-Hilaire, MD;

E. Rasio, MD; and D.K. Nguyen, $M D$

Many conditions may mimic epileptic seizures, including movement disorders, cardiac arrhythmias, psychiatric disorders, or metabolic anomalies. Among these, insulinoma is a rare but curable cause of hypoglycemia often misdiagnosed as epilepsy or a psychiatric disorder. We report three patients with hypoglycemic spells caused by insulinoma. All patients were diagnosed during a video-EEG monitoring session initially performed for the evaluation of intractable epilepsy.

Case reports. Case 1. A 37-year-old woman had a 6-year history of stereotyped paroxysmal episodes of paresthesias of the left arm, spreading to the face and lower limbs, and lasting $\sim 20$ minutes. Episodes could last up to 2 hours and included gait incoordination, mild agitation, emotional lability, and incoherent speech, which was followed by fatigue. Frequency of the attacks gradually increased up to twice a week. The patient was initially diagnosed with partial epilepsy, refractory to carbamazepine. No interictal activity was recorded during a prolonged video-EEG monitoring. Brain MRI was normal, and interictal SPECT revealed bifrontal and right posterior temporal hypoperfusion. A fasting blood glucose level was obtained and showed mild hypoglycemia at $3.2 \mathrm{mmol} / \mathrm{L}$ (normal, 3.8 to $6.1 \mathrm{mmol} / \mathrm{L}$ ). A prolonged fast reproduced a typical attack after 8 hours, along with concomitant severe hypoglycemia $(0.9 \mathrm{mmol} / \mathrm{L})$ and high insulin levels, confirming the diagnosis of insulinoma. An endoscopic ultrasound detected a small $1.5-\mathrm{cm}$ nodular lesion over the pancreatic tail. Surgical removal of the tumor led to full remission of the symptoms. 


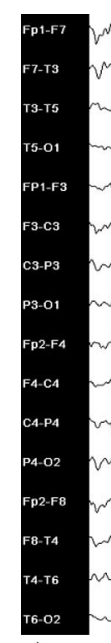

(n)

A

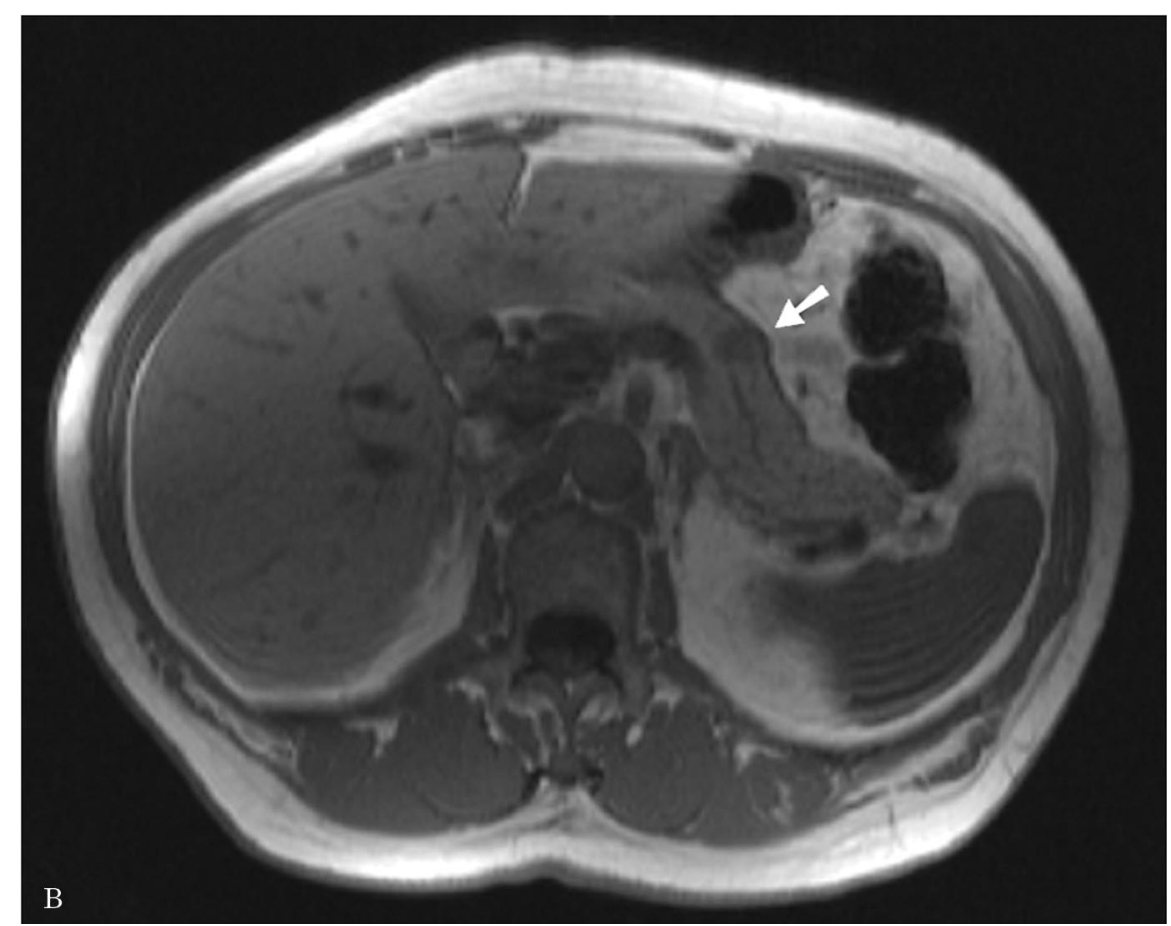

Figure. (A) Mixture of diffuse moderate to high amplitude theta and delta semirhythmic activity during an episode (filters used: high-frequency filter, $35 \mathrm{~Hz}$; low-frequency filter, $1 \mathrm{~Hz}$ ). (B) Abdominal MRI showing a nodular pancreatic lesion.
Case 2. A 44-year-old man had a 2-week history of spells characterized by awkward behavior (crawling, swimming, circling, slamming, and vocalizations), unresponsiveness, and paranoia. Most of these episodes occurred late at night or early morning, lasting from 30 minutes to 6 hours, followed by sleepiness. The attacks increased in intensity and frequency up to a daily basis. These spells were initially misdiagnosed as anxiety disorder and then as complex partial seizures refractory to valproic acid. The EEG, head CT, and glucose levels were normal. A prolonged fast was performed during concomitant video-EEG monitoring. A typical attack was induced after 5 hours associated with ictal diffuse slow waves and significant low blood glucose level $(1.7 \mathrm{mmol} / \mathrm{L})$. Concomitant insulin and C-peptide measures confirmed the inappropriate secretion of insulin. An abdominal CT revealed a $2-\mathrm{cm}$ mass. The patient was cured by a distal pancreatectomy.

Case 3. A 55-year-old woman had a 7-month history of episodic stereotyped confusional spells characterized by psychomotor slowing, unresponsiveness, abnormal posturing, and perseverative unpurposeful behavior. The attacks lasted from a few minutes to 6 hours and occurred up to three times a day despite treatment with carbamazepine and clobazam. Routine serum glucose levels and brain MRI were normal. The patient was admitted to our epilepsy unit with a working diagnosis of psychogenic nonepileptic seizures. No epileptic interictal activity was observed during prolonged video-EEG monitoring. A typical attack was recorded, associated with diffuse slow waves (figure, A), during spontaneous hypoglycemia $(1.4 \mathrm{mmol} / \mathrm{L})$. A prolonged fast was performed and induced a spell after 9 hours with simultaneous low blood glucose $(1.3 \mathrm{mmol} / \mathrm{L})$ and high insulin and C-peptide levels. Abdominal MRI demonstrated a 1-cm lesion in the pancreatic tail (figure, B). The patient was cured by resection of the lesion.

Discussion. Insulinomas may lead to fasting hypoglycemic spells caused by excessive (and time-inappropriate) secretion of insulin. Insulinomas are rare, with 4 cases per 1 million patients each year. ${ }^{1}$ The majority $(>90 \%)$ are benign solitary adenomas, whereas up to $10 \%$ are associated with multiple endocrine neoplasia type I syndromes. ${ }^{1}$ The main challenge of these rare tumors is their recognition. ${ }^{2,3}$ Patients are frequently misdiagnosed with epilepsy or a psychiatric disorder, which was the case in our three patients. It is possible that some of the spells were complex partial seizures secondary to hypoglycemia, although this is doubtful because spells were atypical in semiology, lengthy in duration, unresponsive to anticonvulsant agents, and not associated with ictal epileptic activity during monitoring. In a recent series, median interval from onset of symptoms to diagnosis is 2 years, with a range of 1 month to 30 years. ${ }^{2}$ The diagnostic delay in our patients ranged from 6 months to 8 years. Fortunately, none experienced serious consequences. Late recognition may be caused by many factors, including the rarity of the condition, the lack of specificity of most symptoms, and the blunt of autonomic symptoms from 
deficient counter-regulatory hyperadrenergic responses. ${ }^{4}$ The presence of atypical behavioral spells that are prolonged, recurrent, unresponsive to anticonvulsant agents, and occurring typically after prolonged fast should raise the possibility of hypoglycemia secondary to insulinoma.

The finding of low glucose level in a patient who appears healthy with no coexisting disease warrants further investigation. However, as exhibited in these three patients, a normal result does not exclude the presence of hypoglycemia. When clinically suspected, a supervised prolonged fast (up to 72 hours) under close monitoring should be performed to assess the role of inappropriate secretion of insulin in the genesis of hypoglycemia. A glucose level $<45 \mathrm{mg} / \mathrm{dL}(2.5 \mathrm{mmol} / \mathrm{L})$, a C-peptide level $>0.2 \mathrm{nmol} / \mathrm{L}$, and an insulin level $>6 \mathrm{mU} / \mathrm{mL}(36 \mathrm{pmol} / \mathrm{L})$ confirms the diagnosis of insulinoma. ${ }^{5}$ Localization of tumor could then be assessed by various studies, including abdominal ultrasound, abdominal CT or MRI, selective arteriogram, endoscopic ultrasound, and intraoperative ultrasound. ${ }^{6}$

From the Division of Neurology (Drs. Dion, Cossette, St-Hilaire, and Nguyen) and Endocrinology (Dr. Rasio), Department of Medicine, Centre Hospitalier de l'Université de Montréal, Hôpital Notre-Dame, Montreal, Quebec, Canada.
Received September 30, 2003. Accepted in final form December 15, 2003.

Address correspondence and reprint requests to Dr. Dang Nguyen, Hôpital Notre-Dame, Département de Neurologie, 1560 rue Sherbrooke Est, Montréal, Quebec, H2L 4 M1 Canada; e-mail:d.nguyen@umontreal.ca

Copyright @ 2004 by AAN Enterprises, Inc.

\section{References}

1. Service FJ, McMahon MM, O'Brien PC, Ballard DJ. Functioning insulinoma-incidence, recurrence, and long term survival of patients: a 60 year study. Mayo Clin Proc 1991;66:711-719.

2. Dizon AM, Kowalyk S, Hoogwerf B. Neuroglycopenic and other symptoms in patients with insulinomas. Am J Med 1999;106:307-310.

3. Service FJ, Dale AJ, Elveback LR, Jiang NS. Insulinoma: clinical and diagnostic features of 60 consecutive cases. Mayo Clin Proc 1976;51:417429 .

4. Mitrakou A, Fanelli C, Veneman T, et al. Reversibility of unawareness of hypoglycemia in patients with insulinomas. N Engl J Med 1993;329: 834-839.

5. Service FJ. Diagnostic approach to adults with hypoglycemic disorders. Endocrinol Metab Clin North Am 1999;28:519-532.

6. Modlin IM, Tang LH. Approaches to the diagnosis of gut neuroendocrine tumors: the last word (today). Gastroenterology 1997;112:583-590.

\section{Changes in the appearance of venous sinuses after treatment of disordered intracranial \\ pressure}

Devra B. Baryshnik, MD; and Richard I. Farb, MD

Cerebral angiographic findings in idiopathic intracranial hypertension (IIH) and spontaneous intracranial hypotension (SIH) have been described. Conventional cerebral venography has demonstrated tapered stenoses and filling defects in the transverse sinuses of patients with IIH. ${ }^{1}$ These stenoses have also been found in $>90 \%$ of IIH patients imaged with auto-triggered ellipticcentric-ordered three-dimensional gadolinium-enhanced MR venography (ATECO MRV). ${ }^{2}$ Conventional cerebral angiography demonstrated prominence of intracranial venous structures in patients with SIH. ${ }^{3}$ To date, MRI correlates of these dilated venous channels have not been described. This report discusses the use of ATECO MRV to demonstrate the normalization of venous sinus caliber associated with the resolution of these two disorders of intracranial pressure (ICP).

Case reports. Case 1. A 21-year-old woman was diagnosed with IIH. CSF opening pressure was $32.5 \mathrm{~cm} \mathrm{H}_{2} \mathrm{O}$. Months later, she was admitted to our institution with persistent headache and blurred vision despite maximal doses of acetazolamide. Examination revealed an obese woman with bilateral papilledema. CSF opening pressure remained elevated at $24 \mathrm{~cm} \mathrm{H}_{2} \mathrm{O}$. An MRI of the brain was normal. An ATECO MRV demonstrated the typical stenotic appearance of the dural sinuses (figure, A). ${ }^{2} \mathrm{~A}$ ventriculoperitoneal shunt was inserted, resulting in symptom resolution.

Eighteen months later, the patient had refractory headaches exacerbated by activity, photophobia, nausea, and vomiting. Her medications included acetazolamide $250 \mathrm{mg}$ twice daily and acetaminophen $300 \mathrm{mg} /$ codeine $30 \mathrm{mg}$ two to nine tablets daily for 3 months. CSF pressure was normal $\left(19 \mathrm{~cm} \mathrm{H}_{2} \mathrm{O}\right)$. A CSF nuclear medicine flow study was normal. MRI of the brain demonstrated dural enhancement secondary to the shunt. ATECO MRV revealed normal-appearing venous sinuses with resolution of dural sinus narrowing (figure, B). The patient was diagnosed with transformed migraine secondary to medication overuse.

Case 2. A previously healthy 19-year-old woman with headache and diplopia was transferred to our institution. Her headache was sudden in onset, exacerbated by movement, and severe in the upright position. Examination revealed bilateral abducens palsies. MRI revealed findings typical of intracranial hypotension,

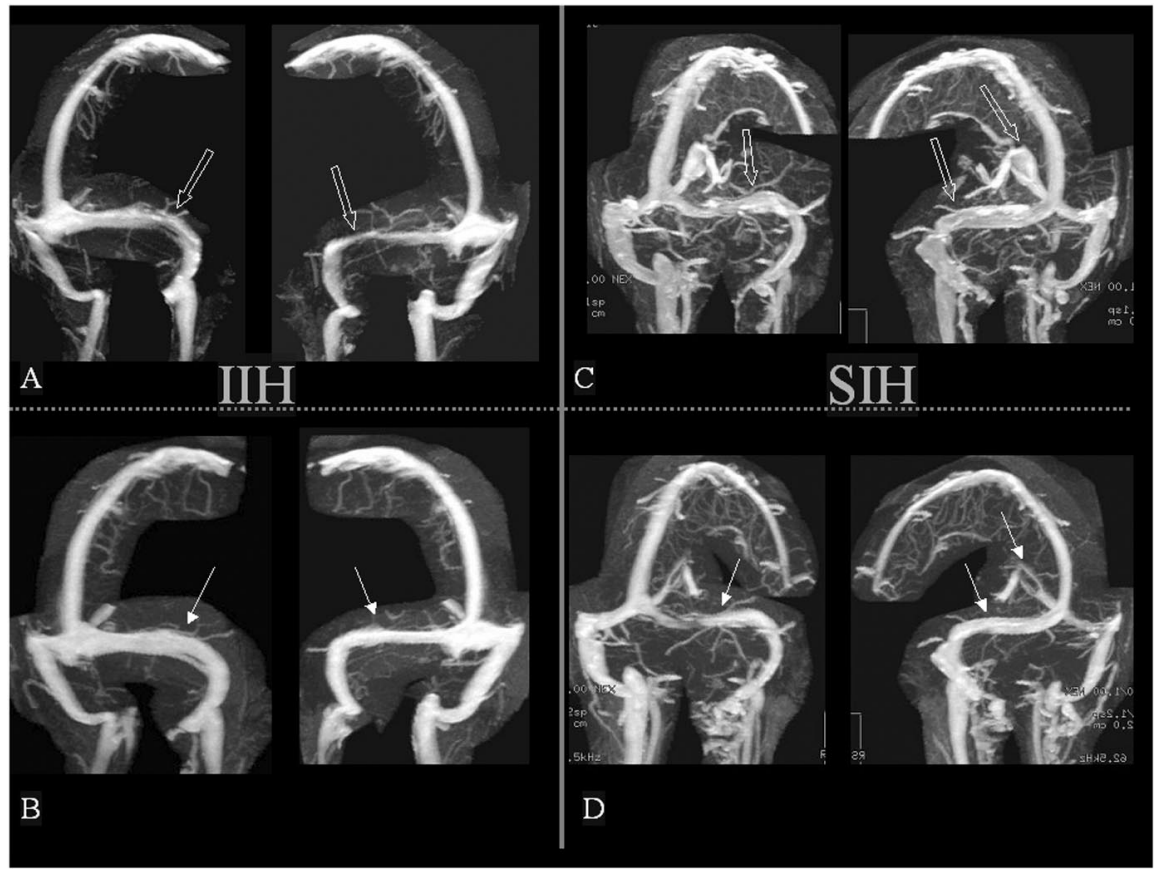

Figure. Paired right anterior oblique and left anterior oblique auto-triggered elliptic-centric-ordered threedimensional gadolinium-enhanced $M R$ (ATECO MR) venogram maximum intensity projection (MIP) images. (A) Patient with idiopathic intracranial hypertension (IIH) before shunt procedure; CSF opening pressure $=24 \mathrm{~cm}$ $\mathrm{H}_{2} \mathrm{O}$. (B) Same patient after shunt procedure; $C S F$ opening pressure $=19 \mathrm{~cm}$ $\mathrm{H}_{2} \mathrm{O}$. (C) Patient with spontaneous intracranial hypotension (SIH) before blood patch procedure. (D) Same patient after blood patch procedure and resolution of symptoms. Open arrows (A and $C$ ) denote abnormal venous sinus caliber before treatment; closed arrows $(B$ and $D$ ) denote normalization of venous sinus caliber post-treatment. 
including inferior displacement of the cerebellar tonsils, flattening of the anterior aspect of the pons, small bilateral subdural hygromas, thickened appearance of the dura, and diffuse pachymeningeal enhancement after gadolinium injection. ${ }^{4}$ An ATECO MRV demonstrated dilatation of the dural venous sinuses (figure, C). A diagnosis of SIH was made. She was treated with a blood patch, and the headache improved. One year later, repeat imaging revealed resolution of the aforementioned stigmata of intracranial hypotension. Specifically, the dural venous sinuses returned to normal caliber (figure, D).

Discussion. These cases demonstrate normalization of venous sinus caliber associated with the resolution of IIH and SIH. The improved imaging and noninvasive nature of ATECO MRV over other venographic techniques have made this possible. ${ }^{5}$ The dynamic changes in venous sinus caliber can be explained using the Monro-Kellie hypothesis. ${ }^{6}$ In IIH, the increase in CSF pressure is compensated for by a decrease in the intracranial blood volume. We propose that the dural venous sinuses act as a volumepressure reservoir that can accommodate these changes. This is seen on ATECO MRV by apparent venous sinus stenosis in patients with IIH. Using the same reasoning, in SIH, a decrease in CSF volume (and thus decrease in pressure) would be accompanied by an increase in the intracranial blood volume. This is seen on ATECO MRV as enlargement of the dural venous sinuses.

Although CSF leakage into extradural space has been accepted as the etiology of SIH, the pathophysiology of IIH has yet to be fully delineated. Cerebral venous manometry in patients with IIH demonstrated a pressure gradient along the transverse sinuses, suggesting the presence of a functional obstruction to venous outflow. ${ }^{1}$ Subsequent investigations demonstrated that removal of 20 to $25 \mathrm{~mL}$ of $\mathrm{CSF}$ corresponded with decrease in central venous sinus pressure, suggesting that the obstruction to venous outflow in IIH is a secondary phenomenon. ${ }^{7}$ These researchers were unable to use venography to demonstrate the normalization of the appearance of the dural venous sinuses with improvement of CSF pressure.

Case 1 provides evidence that the narrowing in the transverse sinuses seen with elevated ICP is reversible when ICP normalizes. This supports the hypothesis that the deformity of the venous sinuses in IIH is a secondary event. Case 2 demonstrates the complementary phenomenon of normalization of venous sinus caliber after clinical and imaging resolution of SIH.

These findings suggest that there is a direct and dynamic relationship between CSF pressure and the appearance of the venous sinuses.

From the Divisions of Neurology (Dr. Baryshnik) and Neuroradiology (Dr. Farb), University of Toronto, University Health Network, Toronto Western Hospital, Toronto, Ontario, Canada.

Received October 29, 2003. Accepted in final form December 15, 2003.

Address correspondence and reprint requests to Dr. Devra B. Baryshnik, University of Toronto, Division of Neurology, 399 Bathurst Street, Toronto, Ontario M5T 2S8, Canada; e-mail: devra.baryshnik@utoronto.ca

Copyright () 2004 by AAN Enterprises, Inc.

\section{References}

1. King JO, Mitchell PJ, Thomson KR, Tress BM. Cerebral venography and manometry in idiopathic intracranial hypertension. Neurology 1995;45: $2224-2228$.

2. Farb RI, Vanek I, Scott JN, et al. Idiopathic intracranial hypertension: the prevalence and morphology of sinovenous stenosis. Neurology 2003 60:1418-1424.

3. Koss SA, Ulmer JL, Hacein-Bey L. Angiographic features of spontaneous intracranial hypotension. AJNR Am J Neuroradiol 2003:24:704-706.

4. Chung SJ, Kim JS, Lee MC. Syndrome of cerebral spinal fluid hypovolemia: clinical and imaging features and outcome. Neurology 2000;55: 1321-1327.

5. Farb R, Scott J, Willinsky R, Montanera W, Wright G, terBrugge K. Intracranial venous system: gadolinium-enhanced three-dimensional MR venography with auto-triggered elliptic centric-ordered sequence-initial experience. Radiology 2003;206:203-209.

6. Mokri B. The Monro-Kellie hypothesis: applications in CSF volume depletion. Neurology 2001;56:1746-1748.

7. King JO, Mitchell PJ, Thomson KR, Tress BM. Manometry combined with cervical puncture in idiopathic intracranial hypertension. Neurology 2002;58:26-30.

\section{Auditory dysfunction in chronic inflammatory demyelinating polyradiculoneuropathy}

\author{
Gerald J.D. Hengstman, MD; Helenius J. Schelhaas, MD; and \\ Machiel J. Zwarts, MD, PhD
}

Cranial nerve involvement, most commonly affecting the facial and oculomotor nerves, has been reported in chronic inflammatory demyelinating polyradiculoneuropathy (CIDP). ${ }^{1,2}$ Symptomatic involvement of the vestibulocochlear nerve has been described at least once but only in the vestibular part of the nerve. ${ }^{1}$

We describe a patient who presented with a relapse of CIDP accompanied by sudden bilateral sensorineural hearing loss. Brainstem auditory evoked potentials (BAEPs) confirmed bilateral retrocochlear dysfunction. Treatment with high-dose prednisone was associated with nearly complete recovery clinically and neurophysiologically.

Case report. A 66-year-old man sought treatment for sudden bilateral hearing loss and paraesthesias of the lower extremities. Ten months earlier, CIDP had been diagnosed based on clinical signs and symptoms (symmetric muscle weakness, sensory ataxia, distal hypesthesia, and absent tendon reflexes), results of nerve conduction studies (i.e., motor distal latency and $\mathrm{F}$ waves of the ulnar and median nerves $>130 \%$ of the upper limit of normal, slowing of nerve conduction velocities in ulnar and peroneal nerves $<70 \%$ of the lower limit of normal, and presence of a conduction block in the peroneal nerve), and CSF analysis (elevated protein without pleocytosis). ${ }^{3}$ He was treated with IV gammaglobulin, which had resulted in a nearly full recovery. At presentation, he did not use any medications besides the occasional use of ciprofloxacin for recurrent cystitis and vitamin B12 supplementation once a month.

At presentation, the patient had sudden bilateral hearing loss and hypesthesia of the feet. He had not noted a change in muscle strength or vertigo. Physical examination showed no abnormalities of the cranial nerves, with the exception of bilateral sensorineural hearing loss. There was areflexia and hypesthesia in the feet. Muscle strength was normal with the exception of slight weakness of the anterior tibial muscles. There was no ataxia. Otoscopy and measurement of pure-tone air and bone conduction thresholds were normal. A pure-tone audiogram revealed a $50-\mathrm{dB}$ HL sensorineural and a 20-dB HL conductive hearing loss on both sides. No BAEP could be elicited at 80 and $90 \mathrm{~dB}$ hearing level (HL) for both ears. At $105 \mathrm{~dB}$ HL, a BAEP was recorded that showed cochlear microphonic potentials, no reproducible peak I and III, delayed peak II, delayed peak IV, and a delayed peak V on the right side and cochlear microphonic potentials, no reproducible peak I and IV, delayed peak II, delayed peak III, and a delayed peak $\mathrm{V}$ on the left side (figure, $\mathrm{A}$ ). The interpeak latency between III and V was slightly increased. Vestibular function tests, including horizontal saccades, smooth pursuit, optokinetic nystagmus, and quantitative rotational testing of the vestibuloocular reflexes, were normal.

The absence of a reproducible peak I on both sides, the increased absolute latencies of all recordable peaks on both sides (even after correction for hearing loss), and the presence of cochlear microphonic potentials suggested a disorder of the cochlear nerve. Because the patient had clinical signs and symptoms suggestive of a relapse of the CIDP, the retrocochlear hearing loss was interpreted as a result of a relapse of the inflammatory demyelinating disorder. Treatment with oral prednisone $(50 \mathrm{mg}$ once a day) was started. Nearly all symptoms resolved within 1 month, and a second pure-tone audiogram demonstrated a residual sensorineural hearing loss of $20 \mathrm{~dB} \mathrm{HL}$ on the left side and $30 \mathrm{~dB} \mathrm{HL}$ on the right side. A second BAEP study showed normal responses on both sides with normal interpeak latencies (figure, B).

Discussion. Cranial nerve involvement is well documented in CIDP and most commonly affects the facial and oculomotor nerves. ${ }^{1,2}$ At least one patient with CIDP and symptomatic involvement of the vestibulocochlear nerve has been described, but only the vestibular part of the eighth cranial nerve was involved. ${ }^{1}$ Our patient is, as far as we know, the first patient described with CIDP and symptomatic involvement of the cochlear part of the vestibulocochlear nerve. 


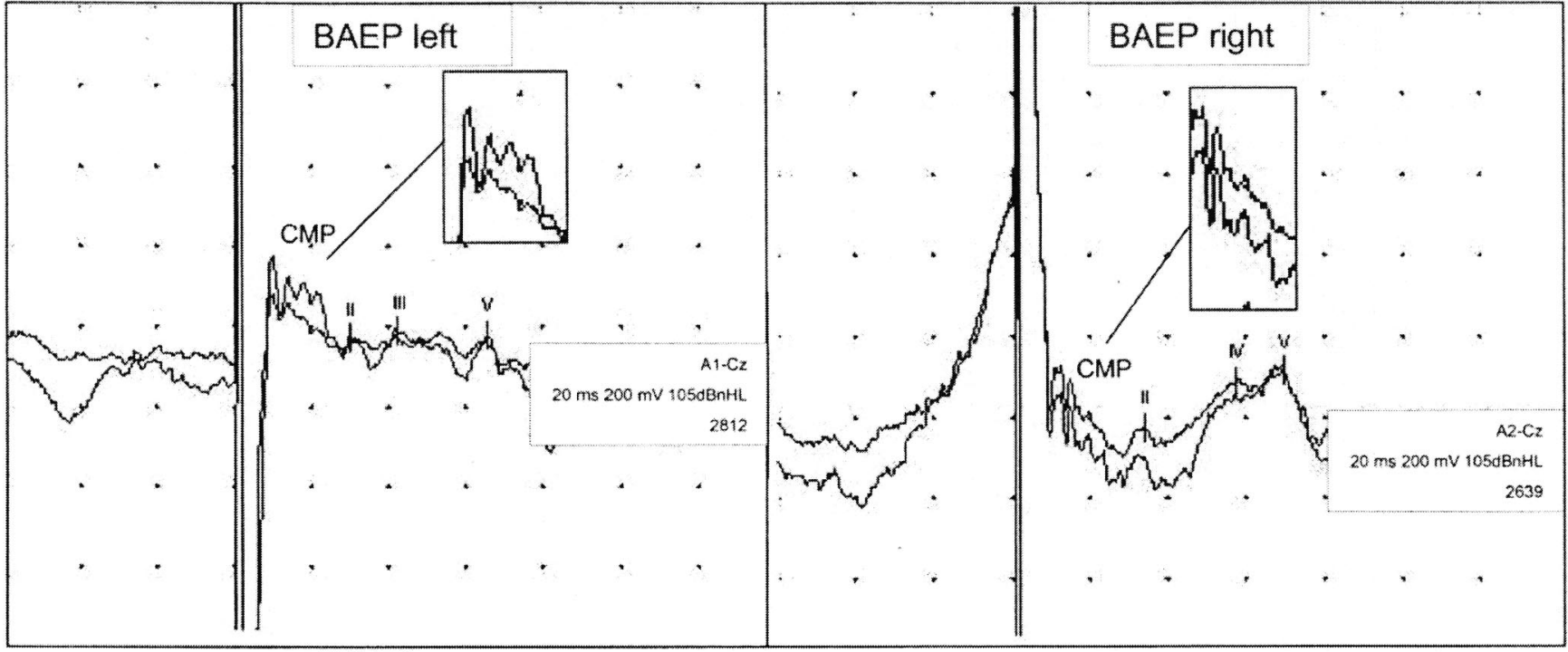

B

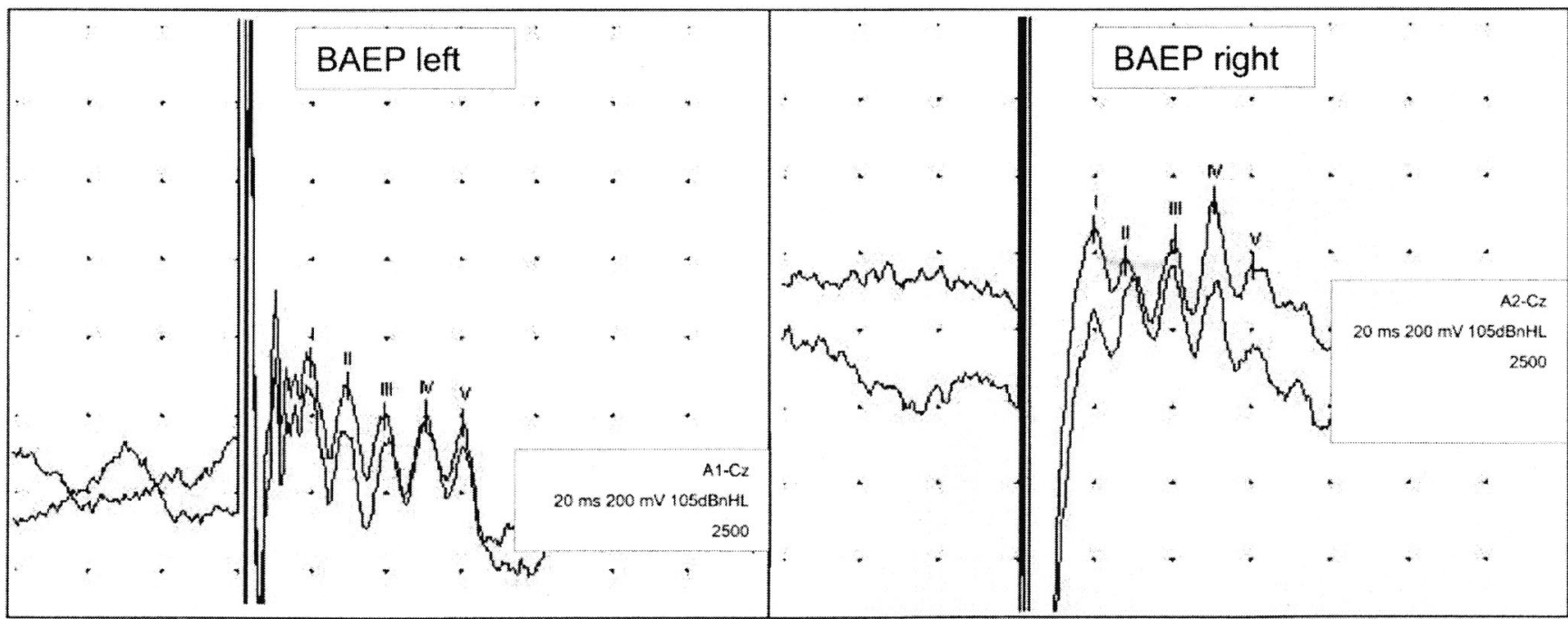

Figure. Brainstem auditory evoked potentials (BAEPs) recorded from A1-Cz and A2-Cz before and after treatment with oral prednisone. (A) BAEP at $105 \mathrm{~dB}$ HL recorded before onset of treatment. Curves are averages from 2,612 (right) and 2,639 (left) recordings. Absolute latencies right side: peak II, $3.44 \mathrm{~ms}$ (normal, 2.45 to 2.95); peak IV, $5.74 \mathrm{~ms}$ (normal, 4.41 to 5.19); and peak V, $6.96 \mathrm{~ms}$ (normal, 4.80 to 5.98). Absolute latencies left side: peak II, $3.00 \mathrm{~ms}$; peak III, $4.26 \mathrm{~ms}$ (normal, 3.34 to 3.90); and peak V, $6.58 \mathrm{~ms}$. (B) BAEP at $95 \mathrm{~dB}$ HL recorded after treatment with oral prednisone. Curves are averages from 2,500 recordings. Absolute latencies right side: peak I, $1.98 \mathrm{ms;}$ peak II, $2.78 \mathrm{~ms}$; peak III, 4.00 ms; peak IV, $5.02 \mathrm{~ms}$; and peak V, $6.08 \mathrm{~ms}$. Absolute latencies left side: peak I, $1.98 \mathrm{~ms}$; peak II, $2.96 \mathrm{~ms}$; peak III, 3.98 $\mathrm{ms}$; peak IV, $5.06 \mathrm{~ms}$; and peak $\mathrm{V}, 6.08 \mathrm{~ms}$. CMP = cochlear microphonic potentials.

Abnormal BAEPs in patients with CIDP have been described, but none of these patients had auditory symptoms. ${ }^{4-6}$ Moreover, in these studies a second BAEP investigation was not performed after management of the CIDP, thus leaving unanswered the question whether BAEP abnormalities are reversible, as might be expected in an immune-mediated demyelinating disorder. The results of the second BAEP study in our patient clearly showed that the BAEP abnormalities were reversible.

From the Neuromuscular Centre Nijmegen, Department of Neurology, Uni versity Medical Centre Nijmegen, The Netherlands.

Received August 28, 2003. Accepted in final form December 17, 2003.
Address correspondence and reprint requests to Dr. G.J.D. Hengstman, Neuromuscular Centre Nijmegen, Department of Neurology, University Medical Centre Nijmegen, P.O. Box 9101, 6500 HB Nijmegen, The Netherlands; e-mail: g.hengstman@neuro.umcn.nl

Copyright (C) 2004 by AAN Enterprises, Inc.

\section{References}

1. Frohman EM, Tusa R, Mark AS, Cornblath DR. Vestibular dysfunction in chronic inflammatory demyelinating polyneuropathy. Ann Neurol 1996;39:529-535.

2. Dalakas MC, Engel WK. Chronic relapsing (dysimmune) polyneuropathy: pathogenesis and treatment. Ann Neurol 1981;9(suppl):134-145. 
3. Franssen H, Vermeulen M, Jennekens FGI. Chronic inflammatory neuropathies. In: Emery AEH, ed. Diagnostic Criteria for Neuromuscular Disorders. London: Royal Society of Medicine Press, 1997:53-59.

4. Pakalnis A, Drake Jr ME, Barohn RJ, Chakeres DW, Mendell JR. Evoked potentials in chronic inflammatory demyelinating polyneuropathy. Arch Neurol 1988;45:1014-1016.
5. Gigli GL, Carlesimo A, Valente M, et al. Evoked potentials suggest cranial nerves and CNS involvement in chronic relapsing polyradiculoneuropathy. Eur Neurol 1989;29:145-149.

6. Ohtake T, Komori T, Hirose K, Tanabe H. CNS involvement in Japanese patients with chronic inflammatory demyelinating polyradiculoneuropathy. Acta Neurol Scand 1990;81:108-112.

\section{ACTIVATE YOUR ONLINE SUBSCRIPTION}

At www.neurology.org, subscribers can now access the full text of the current issue of Neurology and back issues to 1999. Select the "Login instructions" link that is provided on the Help screen. Here you will be guided through a step-by-step activation process.

Neurology online offers:

- Access to journal content in both Adobe Acrobat PDF or HTML formats

- Links to PubMed

- Extensive search capabilities

- Complete online Information for Authors

- Examinations on designated articles for CME credit

- Access to in-depth supplementary scientific data 